\title{
A Heteronuclear Bidentate Lewis Acid as a Phosphorescent Fluoride Sensor.
}

Mohand Melaimi and François P. Gabbaï*

Chemistry Department

Texas A\&M University 3255 TAMU

College Station, Texas 77843-3255

Fax: (1) 979-845-4719

E-mail: gabbai@mail.chem.tamu.edu

Supplementary information 


\section{Experimental part}

$\underline{\text { General Considerations. }}$

Due to the toxicity of the mercury compounds discussed in these studies, great caution should be exercised when synthesizing and handling 2 and its anionic derivatives. Tetrakis(THF)lithium dimesityl-1,8-naphthalenediylborate (Hoefelmeyer, J. D.; Gabbaï, F. P. Organometallics 2002, 21, 982) and pentafluorophenylmercury chloride (Tschinkl, M. Schier, A. Riede, J. Gabbaï F. P. Organometallics 1999, 18, 2040) were synthesized by following published procedures. $\mathrm{Me}_{3} \mathrm{SiF}_{2} \mathrm{~S}\left(\mathrm{NMe}_{2}\right)_{3}$ and trimethylchlorosilane were purchased from Aldrich and used as received. Solvents were dried by reflux under $\mathrm{N}_{2}$ over the appropriate drying agents, and freshly distilled prior to use. Dichloromethane was dried over $\mathrm{CaH}_{2}$. Diethylether and THF were dried over $\mathrm{Na} / \mathrm{K}$. Air-sensitive compounds were handled under $\mathrm{N}_{2}$ atmosphere using standard Schlenk and glovebox techniques. UV-vis spectra were recorded on a JASCO V-530 spectrophotometer. Elemental analyses were performed at Atlantic Microlab (Norcross, GA). The luminescence spectra were recorded with a SLM/AMINCO, Model 8100 spectrofluorometer equipped with a xenon lamp. All melting points were measured on samples in sealed capillaries and are uncorrected.

\section{NMR spectroscopy}

NMR spectra were recorded on a Varian Unity Inova 400 FT NMR spectrometer (399.63 $\mathrm{MHz}$ for ${ }^{1} \mathrm{H}, 376.03 \mathrm{MHz}$ for ${ }^{19} \mathrm{~F}, 128.22 \mathrm{MHz}$ for ${ }^{11} \mathrm{~B}, 100.50 \mathrm{MHz}$ for ${ }^{13} \mathrm{C}, 75.52$ for

${ }^{199} \mathrm{Hg}$ ) by using internal deuterium lock. Chemical shifts $\delta$ are given in ppm, and are 
referenced against external $\mathrm{Me}_{4} \mathrm{Si}\left({ }^{1} \mathrm{H},{ }^{13} \mathrm{C}\right), \mathrm{BF}_{3} \cdot \mathrm{Et}_{2} \mathrm{O}\left({ }^{11} \mathrm{~B}\right), \mathrm{CFCl}_{3}\left({ }^{19} \mathrm{~F}\right)$, and $\mathrm{HgMe}_{2}$ $\left({ }^{199} \mathrm{Hg}\right)$.

\section{$\underline{\text { Crystallography }}$}

The crystallographic measurements were performed using a Siemens SMART-CCD area detector diffractometer, with a graphite-monochromated $\mathrm{Mo}-\mathrm{K}_{\alpha}$ radiation $(\lambda=0.71069$ $\AA$ ). Specimens of suitable size and quality were selected and mounted onto glass fiber with apiezon grease. The structures were solved by direct methods, which successfully located most of the non-hydrogen atoms. Subsequent refinement on $F^{2}$ using the SHELXTL/PC package (version 5.1) allowed location of the remaining non-hydrogen atoms. Further crystallographic details can be found in table 1.

\section{$\underline{\text { Synthesis and characterization }}$}

1-(dimesitylboron)-8-(pentafluorophenylmercury)naphthalenediyl (2). A solution of pentafluoromecury chloride $(360 \mathrm{mg}, 0.89 \mathrm{mmol})$ in THF $(3 \mathrm{~mL})$ was added to a solution of tetrakis(THF)lithium dimesityl-1,8-naphthalenediylborate $(600 \mathrm{mg}, 0.89 \mathrm{mmol})$ in THF $(10 \mathrm{~mL})$ at $0^{\circ} \mathrm{C}$. The mixture was stirred overnight at room temperature. The solvent were removed under reduced pressure and the product was extracted with hexane $(3 \times 10$ $\mathrm{mL}$ ). Following filtration and evaporation of the solvent, the yellow solid was washed with acetonitrile $(4 \times 5 \mathrm{~mL})$ and dried under vacuum to yield a pale yellow powder (480 mg : 72\%). mp. $235^{\circ} \mathrm{C} .{ }^{1} \mathrm{H}$ NMR $\left(\mathrm{CDCl}_{3}\right): 1.44$ (br s, $3 \mathrm{H}$, Mes-CH ), 1.67 (br s, 3H, Mes$\mathrm{CH}_{3}$ ), 1.89 (br s, 3H, Mes- $\mathrm{CH}_{3}$ ), 2.14-2.42 (m, 9H, Mes- $\mathrm{CH}_{3}$ ), 6.37 (br s, $1 \mathrm{H}$, Mes-CH), 6.83 (br s, 3H, Mes-CH), 7.37-7.44 (m, 2H, CH), 7.48-7.60 (m, 2H, CH), 7.86 (dd, $1 \mathrm{H}$, 
$\left.{ }^{3} J_{\mathrm{H}-\mathrm{H}}=8.0 \mathrm{~Hz},{ }^{4} J_{\mathrm{H}-\mathrm{H}}=1.1 \mathrm{~Hz}, \mathrm{CH}\right), 7.98\left(\mathrm{dd}, 1 \mathrm{H},{ }^{3} J_{\mathrm{H}-\mathrm{H}}=8.0 \mathrm{~Hz},{ }^{4} J_{\mathrm{H}-\mathrm{H}}=1.2 \mathrm{~Hz}, \mathrm{CH}\right)$.

${ }^{13} \mathrm{C}$ NMR $\left(\mathrm{CDCl}_{3}\right): 21.34,22.54,23.83,25.23\left(\mathrm{CH}_{3}-\mathrm{Mes}\right), 125.53,125.75,130.48$ 133.74, 135.18, 137.29 (CH-Np), 127.63 (br s), 128.64 (br s), 130.36 (br s), 130.72 (br s) (CH-Mes), 134.76, 142.42, 151.50, (CF). 136.89 (br s), 138.65 (br s), 138.89 (br s), 139.34 (br s), 139.81 (br s), 143.50 (br s), 143.88 (br s), 146.22, 146.43, 146.96, 148.51, 148.70, 163.54 (quaternary $\mathrm{C}, \mathrm{CB}$ and $\mathrm{CHg}) .{ }^{19} \mathrm{~F}$ NMR $\left(\mathrm{CDCl}_{3}\right):-116.8\left(\mathrm{~m}, 2 \mathrm{~F},{ }^{3} J_{\mathrm{F}-\mathrm{Hg}}=\right.$ $\left.499 \mathrm{~Hz}, \mathrm{~F}_{\text {ortho }}\right),-152.5\left(\mathrm{t}, 1 \mathrm{~F},{ }^{3} \mathrm{~J}_{\mathrm{F}-\mathrm{F}}=19.7 \mathrm{~Hz}, \mathrm{~F}_{\text {para }}\right),-159.09\left(\mathrm{~m}, 2 \mathrm{~F}, \mathrm{~F}_{\text {meta }}\right) .{ }^{199} \mathrm{Hg} \mathrm{NMR}$ $\left(\mathrm{CDCl}_{3}\right):-741.9\left(\mathrm{tt},{ }^{3} J_{\mathrm{Hg}-\mathrm{F}}=499 \mathrm{~Hz},{ }^{4} J_{\mathrm{Hg}-\mathrm{F}}=165 \mathrm{~Hz}\right) .{ }^{11} \mathrm{~B} \mathrm{NMR}\left(\mathrm{CDCl}_{3}\right):+72$. Anal. Calcd for $\mathrm{C}_{34} \mathrm{H}_{28} \mathrm{BF}_{5} \mathrm{Hg}$ : C, 54.96; H, 3.80. Found: C 54.92; H 3.75.

$\left[2 \cdot \mu_{2}-\mathbf{F}\right]^{-}\left[\mathbf{S}\left(\mathbf{N M e}_{2}\right)_{3}\right]^{+}:$To a solution of $\mathbf{2}(100 \mathrm{mg}, 0.13 \mathrm{mmol})$ in $\mathrm{CHCl}_{3}(2 \mathrm{~mL})$ was added one equivalent of TASF ( $37 \mathrm{mg}, 0.13 \mathrm{mmol}$ ) at room temperature. The mixture is stirred at room temperature for 10 minutes and stored at $-18^{\circ} \mathrm{C}$ overnight to induce the crystallization of $\left[2 \cdot \mu_{2}-\mathrm{F}\right]^{-}\left[\mathrm{S}\left(\mathrm{NMe}_{2}\right)_{3}\right]^{+}$. After filtration and washing with hexane $(2 \mathrm{~mL})$ and ether $(2 \mathrm{~mL})$, the product was obtained as colorless crystals ( $\mathrm{m}=110 \mathrm{mg}, 89 \%)$. $\mathrm{mp}$. $215{ }^{\circ} \mathrm{C}(\mathrm{dec}) .{ }^{1} \mathrm{H}$ NMR $\left(\mathrm{CD}_{3} \mathrm{CN}\right.$ ): 1.67 (br s, $6 \mathrm{H}$, Mes- $\mathrm{CH}_{3}$ ), 1.83 (br s, $3 \mathrm{H}$, Mes-CH $\mathrm{CH}_{3}$, 2.00 (br s, 3H, Mes- $\mathrm{CH}_{3}$ ), 2.16 (m, 6H, Mes-CH $\mathrm{CH}_{3}$ ), 2.84 (s, 18H, N-CH$H_{3}$ ), 6.32-6.64 (br s, $4 \mathrm{H}$, Mes-CH), $7.08\left(\mathrm{t}, 1 \mathrm{H},{ }^{3} J_{\mathrm{H}-\mathrm{H}}=7.5 \mathrm{~Hz}\right), 7.19\left(\mathrm{dd}, 1 \mathrm{H},{ }^{3} J_{\mathrm{H}-\mathrm{H}}=7.5 \mathrm{~Hz},{ }^{4} J_{\mathrm{H}-\mathrm{H}}=1.4 \mathrm{~Hz}\right)$, $7.30\left(\mathrm{~d}, 2 \mathrm{H},{ }^{3} J_{\mathrm{H}-\mathrm{H}}=5.1 \mathrm{~Hz}\right), 7.54\left(\mathrm{dd}, 1 \mathrm{H},{ }^{3} J_{\mathrm{H}-\mathrm{H}}=7.5 \mathrm{~Hz},{ }^{4} J_{\mathrm{H}-\mathrm{H}}=1.4 \mathrm{~Hz}\right), 7.69(\mathrm{t}, 1 \mathrm{H}$, $\left.{ }^{3} J_{\mathrm{H}-\mathrm{H}}=5.1 \mathrm{~Hz}\right) .{ }^{13} \mathrm{C} \mathrm{NMR}\left(\mathrm{CD}_{3} \mathrm{CN}\right): 20.04(\mathrm{Br} \mathrm{s}), 23.65(\mathrm{Br} \mathrm{s}), 24.44(\mathrm{Br} \mathrm{s})\left(\mathrm{CH}_{3}-\mathrm{Mes}\right)$, $37.99\left(\mathrm{~N}-\mathrm{CH}_{3}\right), 123.10,124.78,126.35,129.40,132.81,135.88(\mathrm{CH}-\mathrm{Np}), 127.91$ (Br s), 128.33 (Br s), 128.92 (Br s) (CH-Mes), 131.90 (br s), 134.29 (br s), 135.53, 136.46, $138.24,138.68,138.94,140.59$ (br s), 142.50 (br s), 145.54, 145.92, 146.32, 148.51, 
149.11, 150.12 (br s), 156.26 (br s), 161.18 (br s), 165.87 (CF, quaternary C, $C \mathrm{~B}$ and CHg). ${ }^{19} \mathrm{~F}$ NMR $\left(\mathrm{CD}_{3} \mathrm{CN}\right):-117.1\left(\mathrm{~m}, 2 \mathrm{~F},{ }^{2} J_{\mathrm{F}-\mathrm{Hg}}=384.8 \mathrm{~Hz}, \mathrm{~F}_{\text {ortho }}\right),-159.0\left(\mathrm{t}, 1 \mathrm{~F},{ }^{3} J_{\mathrm{F}-\mathrm{F}}=\right.$ 19.2 Hz, $\left.\mathrm{F}_{\text {para }}\right),-163.5$ (m, 2F, $\left.\mathrm{F}_{\text {meta }}\right),-164.3$ (m, 1F, $\left.\mu^{2}-\mathrm{F}\right) .{ }^{199} \mathrm{Hg} \mathrm{NMR}\left(\mathrm{CD}_{3} \mathrm{CN}\right):-811.8$ $\left(\mathrm{dtt},{ }^{1} J_{\mathrm{Hg}-\mathrm{F}}=135.2 \mathrm{~Hz},{ }^{3} J_{\mathrm{Hg}-\mathrm{F}}=387.1 \mathrm{~Hz},{ }^{4} J_{\mathrm{Hg}-\mathrm{F}}=65.8 \mathrm{~Hz}\right) .{ }^{11} \mathrm{~B} \mathrm{NMR}\left(\mathrm{CD}_{3} \mathrm{CN}\right):+8.5$ Anal. Calcd for $\mathrm{C}_{40} \mathrm{H}_{46} \mathrm{BF}_{6} \mathrm{HgN}_{3} \mathrm{~S}$ : C, 51.87; H, 5.01. Found: C 51.48; H 4.91. MS (ESI) $m / z=763.135$ [2-F] . CV (DMSO): reversible wave at $1.965 \mathrm{eV}$ relatice to $\mathrm{Fc} / \mathrm{Fc}^{+}$. 
UV-Vis spectrum of 2 and $\left[2-\mu^{2}-\mathrm{F}\right]^{-}\left[\mathrm{nBu} u_{4} \mathrm{~N}\right]^{+}$- Titration of 2 with $\mathrm{F}^{-}$

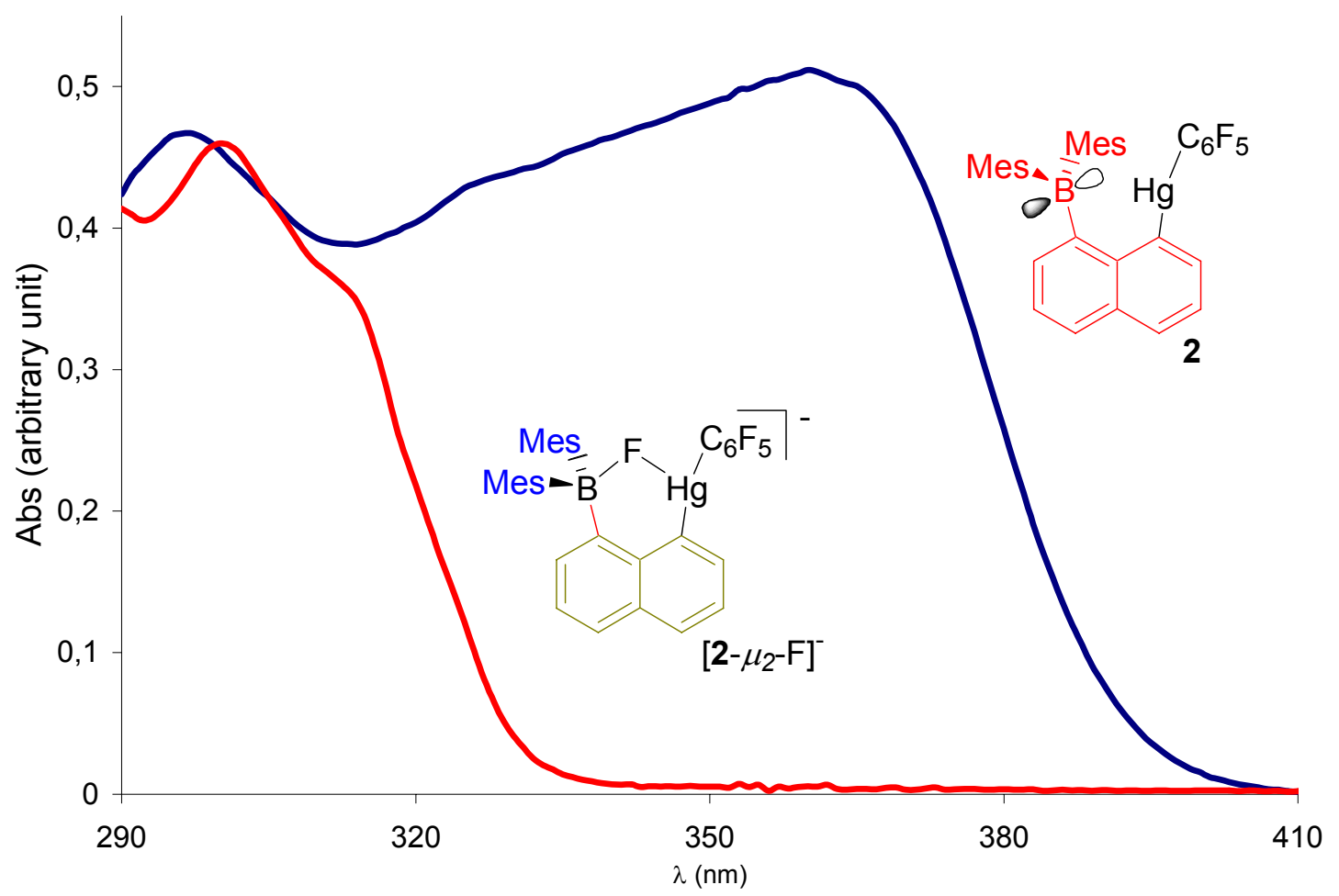

Figure SI- 1: UV-Visible spectrum of 2 and $\left[2-\mu^{2}-\mathrm{F}\right]^{-} \cdot\left[\mathrm{nBu}_{4} \mathrm{~N}\right]^{+}$.

\section{Derivation of the fluoride titration isotherm}

In the following equations $\mathrm{pFBHg}=\mathbf{2}$ and $\mathrm{pFBHgF}=\left[\mathbf{2} \cdot \mu^{2}-\mathrm{F}\right]^{-}$.

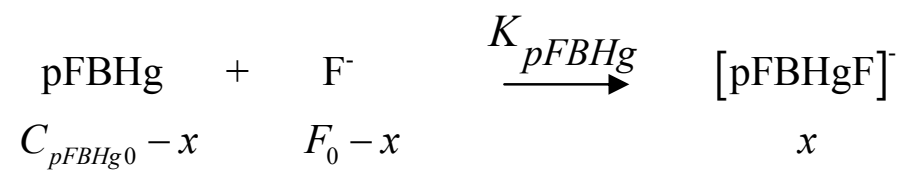

Equation SI- 1: Chemical distribution after addition of fluoride to a solution of 2. 


$$
\begin{aligned}
& K_{p F B H g}=\frac{[p F B H g]}{[p F B H g F] *\left[F^{-}\right]}=\frac{x}{\left(C_{p F B H g 0}-x\right) *\left(F_{0}^{-}-x\right)} \\
& \Rightarrow x^{2}-x\left(C_{p F B H g 0}+F_{0}^{-}+\frac{1}{K_{p F B H g}}\right)+C_{p F B H g 0} * F_{0}^{-}=0 \\
& \Rightarrow x=\frac{A-\sqrt{A^{2}-4 * C_{p F B H g} * F_{0}^{-}}}{2} \quad \text { with } A=C_{p F B H g 0}+F_{0}^{-}+\frac{1}{K_{p F B H g}}
\end{aligned}
$$

Equation SI- 2: Relationship between the observed absorption with respect to the amount of fluoride added and to the binding constant $\mathrm{K}_{\mathrm{pFBHg}}$.

\section{Titration in THF}

A solution of $2\left(3 \mathrm{~mL}, 510^{-5} \mathrm{M}\right.$, THF) was placed in the cell and was titrated with incremental amounts of fluoride by addition of a solution of $\mathrm{nBu}_{4} \mathrm{NF}$ in THF $\left(5.8210^{-3}\right.$ M). The absorption was monitored at $\lambda_{\max }=361 \mathrm{~nm}(\varepsilon=10200)$.

\begin{tabular}{|c|c|c|c|}
\hline $\mathrm{C}_{\text {Fluoride }}$ & $\mathrm{Abs}_{(361 \mathrm{~nm})}$ & $\mathrm{C}_{\text {Fluoride }}$ & $\mathrm{Abs}_{(361 \mathrm{~nm})}$ \\
\hline $0.00 \mathrm{E}+00$ & 0.51022 & $3.61 \mathrm{E}-05$ & 0.13821 \\
\hline $2.42 \mathrm{E}-06$ & 0.48784 & $3.85 \mathrm{E}-05$ & 0.11064 \\
\hline $4.84 \mathrm{E}-06$ & 0.45853 & $4.09 \mathrm{E}-05$ & 0.08489 \\
\hline $7.26 \mathrm{E}-06$ & 0.43579 & $4.33 \mathrm{E}-05$ & 0.05932 \\
\hline $9.68 \mathrm{E}-06$ & 0.41097 & $4.57 \mathrm{E}-05$ & 0.03284 \\
\hline $1.21 \mathrm{E}-05$ & 0.38955 & $4.81 \mathrm{E}-05$ & 0.01376 \\
\hline $1.45 \mathrm{E}-05$ & 0.36446 & $5.04 \mathrm{E}-05$ & 0.00443 \\
\hline $1.69 \mathrm{E}-05$ & 0.34091 & $5.28 \mathrm{E}-05$ & 0.00377 \\
\hline $1.93 \mathrm{E}-05$ & 0.31701 & $5.52 \mathrm{E}-05$ & 0.00579 \\
\hline $2.17 \mathrm{E}-05$ & 0.29065 & $5.76 \mathrm{E}-05$ & 0.0055 \\
\hline $2.41 \mathrm{E}-05$ & 0.26501 & $6.00 \mathrm{E}-05$ & 0.00513 \\
\hline $2.65 \mathrm{E}-05$ & 0.23971 & $6.23 \mathrm{E}-05$ & 0.00585 \\
\hline $2.89 \mathrm{E}-05$ & 0.2119 & $6.47 \mathrm{E}-05$ & 0.00732 \\
\hline $3.13 \mathrm{E}-05$ & 0.18763 & $6.71 \mathrm{E}-05$ & 0.00599 \\
\hline $3.37 \mathrm{E}-05$ & 0.16385 & & \\
\hline
\end{tabular}

Table SI- 1: Absorbance of a solution of 2 (THF) after successive additions of fluoride. 


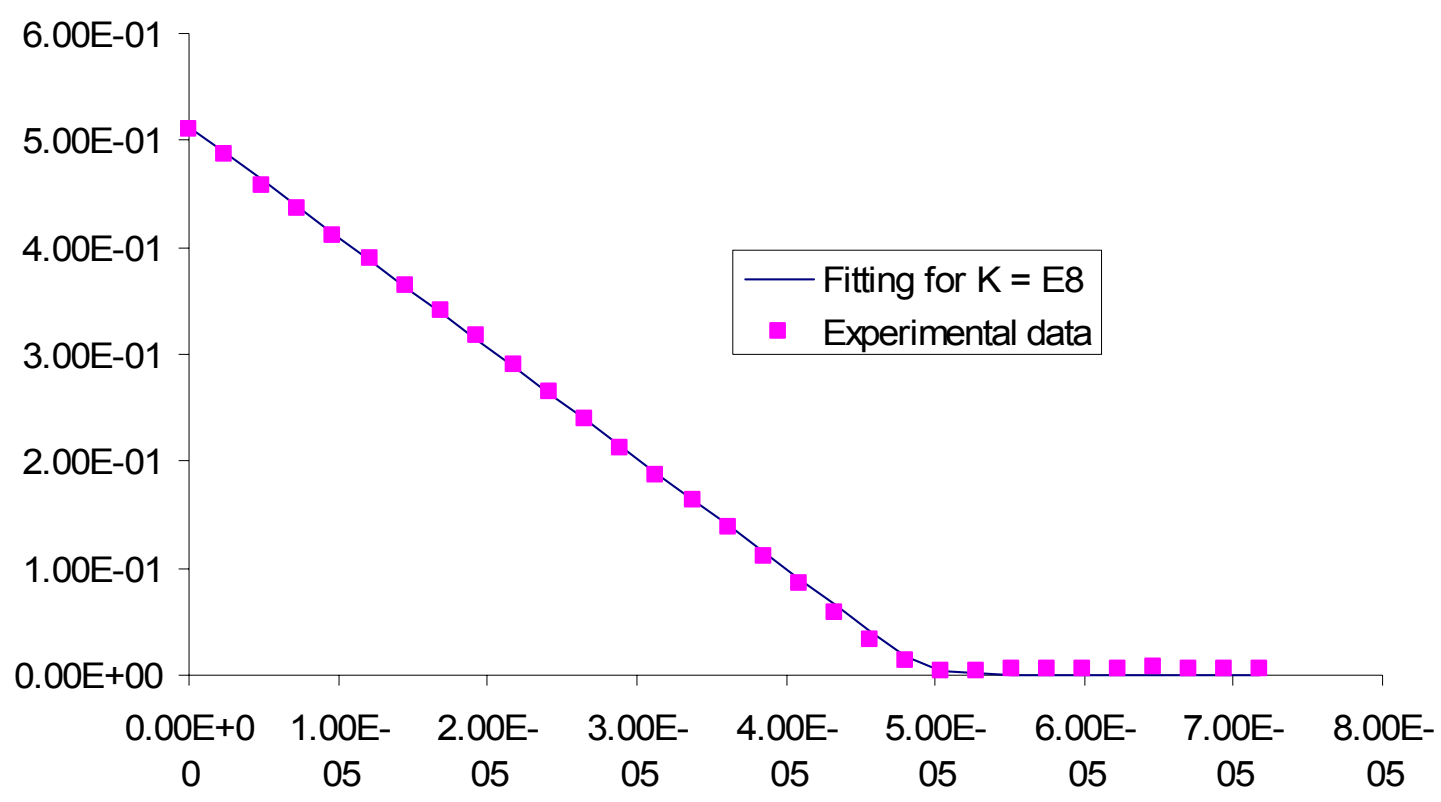

Figure SI- 2: Absorbance of a solution of 2 (THF) vs. fluoride concentration (M)

The experimental data obtained indicated that the binding constant is higher than $10^{8} \mathrm{M}^{-1}$ in THF.

$\underline{\text { Determination of the fluoride binding constant of trimesitylborane in THF- }{ }_{2}} \underline{\underline{O}(90 / 10)}$

$$
\begin{aligned}
& \mathrm{Mes}_{3} \mathrm{~B}+\mathrm{F}^{-} \stackrel{K_{\mathrm{Mes}_{3} B}}{\longrightarrow} \quad\left[\mathrm{Mes}_{3} \mathrm{BF}\right] \\
& C_{0}-x \quad C_{0}-x \quad x \\
& \text { with } C_{0}=C_{\text {Mes } B 0_{3}}=F_{0}^{-}=0.163 \mathrm{M} \text {. } \\
& K_{\text {Mes }_{3} B}=\frac{\left[\mathrm{Mes}_{3} \mathrm{BF}\right]^{-}}{\left[\mathrm{Mes}_{3} \mathrm{~B}\right] *\left[\mathrm{~F}^{-}\right]}=\frac{x}{\left(C_{0}-x\right)^{2}}
\end{aligned}
$$

Equation SI- 3: Chemical distribution after addition of fluoride to a solution of $\mathrm{Mes}_{3} \mathrm{~B}$ and TBAF.

$60 \mathrm{mg}$ of trimesitylborane $(1.63 \mathrm{mmol})$ and $51 \mathrm{mg}$ of TBAF- $3 \mathrm{H}_{2} \mathrm{O}(1.63 \mathrm{mmol}, 1 \mathrm{eq})$ were dissolved in $1 \mathrm{~mL}$ of a THF- $\mathrm{H}_{2} \mathrm{O}(90 / 10)$ solution. The ${ }^{19} \mathrm{~F}$ NMR $(400 \mathrm{MHz})$ was recorded, showing two signals at $-119.3 \mathrm{ppm}$ and $-151.7 \mathrm{ppm}$ for free fluoride anion and trimesitylborane fluoride adduct respectively. 


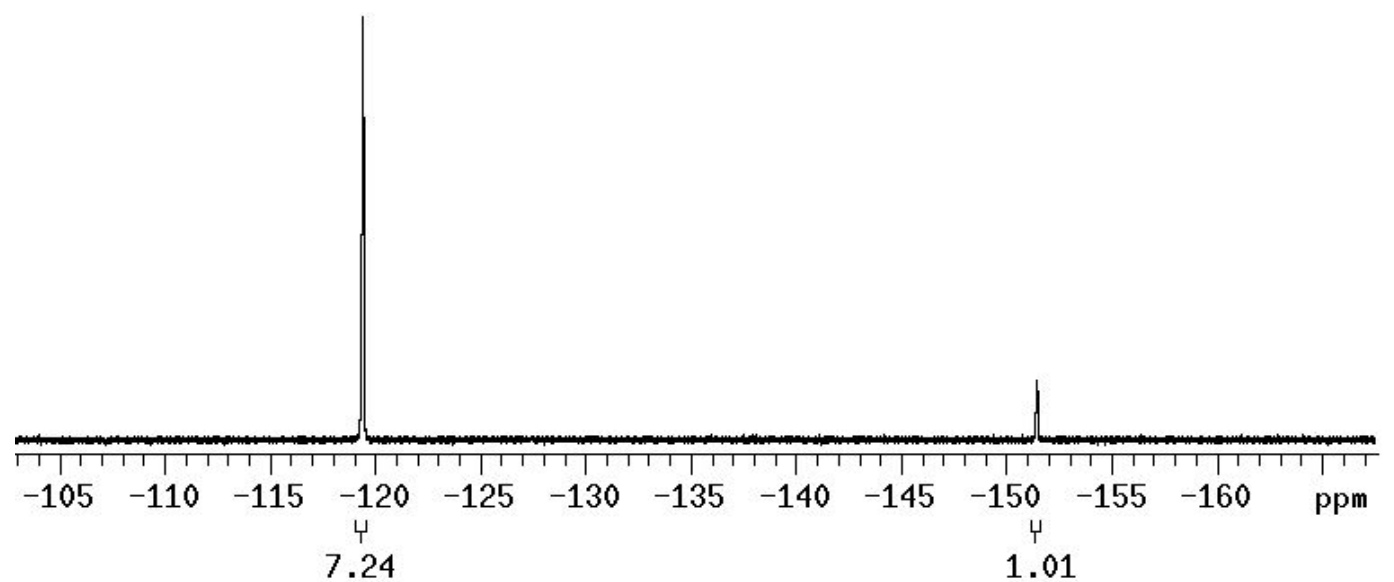

Figure SI- 3: ${ }^{19} \mathrm{~F}$ NMR of a $0.163 \mathrm{M}$ solution of $\mathrm{BMes}_{3}$ and TBAF in THF- $\mathrm{H}_{2} \mathrm{O}(90 / 10)$.

The relative integration gives $\mathrm{x}=1.9910^{-2} \mathrm{M}$, and $\mathrm{C}_{0}-\mathrm{X}=0.143 \mathrm{M}$. Therefore the binding constant of trimesitylborane is $\mathrm{K}_{M e s 3 B}=1( \pm 0.3) \mathrm{M}^{-1}$.

\section{$\underline{\text { Titration in a THF- } \mathrm{H}_{2}} \underline{\mathrm{O}(90 / 10)}$}

A solution of $2\left(3 \mathrm{~mL}, 4.510^{-5} \mathrm{M}\right.$, THF- $\mathrm{H}_{2} \mathrm{O} 90 / 10$ (vol.)) was placed in the cell and was titrated with incremental amounts of fluoride by addition of a solution of $\mathrm{nBu}_{4} \mathrm{NF}$ in THF $\left(5.710^{-3} \mathrm{M}\right)$. The absorption was monitored at $\lambda_{\max }=361 \mathrm{~nm}(\varepsilon=9850)$.

\begin{tabular}{|c|c|c|c|}
\hline $\mathrm{C}_{\text {Fluoride }}$ & $\mathrm{Abs}_{(361 \mathrm{~nm})}$ & $\mathrm{C}_{\text {Fluoride }}$ & $\mathrm{Abs}_{(361 \mathrm{~nm})}$ \\
\hline $0.00 \mathrm{E}+00$ & 0.45047 & $5.18 \mathrm{E}-05$ & 0.24579 \\
$4.75 \mathrm{E}-06$ & 0.43114 & $5.64 \mathrm{E}-05$ & 0.21995 \\
$9.48 \mathrm{E}-06$ & 0.40972 & $7.50 \mathrm{E}-05$ & 0.1829 \\
$1.42 \mathrm{E}-05$ & 0.38949 & $9.34 \mathrm{E}-05$ & 0.15881 \\
$1.89 \mathrm{E}-05$ & 0.3701 & $1.12 \mathrm{E}-04$ & 0.13525 \\
$2.37 \mathrm{E}-05$ & 0.34852 & $1.30 \mathrm{E}-04$ & 0.11735 \\
$2.84 \mathrm{E}-05$ & 0.31669 & $1.48 \mathrm{E}-04$ & 0.10578 \\
$3.31 \mathrm{E}-05$ & 0.30459 & $1.66 \mathrm{E}-04$ & 0.09523 \\
$3.77 \mathrm{E}-05$ & 0.28971 & $1.84 \mathrm{E}-04$ & 0.08509 \\
$4.24 \mathrm{E}-05$ & 0.26564 & $2.02 \mathrm{E}-04$ & 0.07974 \\
$4.71 \mathrm{E}-05$ & 0.25625 & & \\
\hline
\end{tabular}

Table SI- 2: Absorbance of a solution of 2 (THF- $\mathrm{H}_{2} \mathrm{O}:$ 90/10) after successive addition of fluoride. 


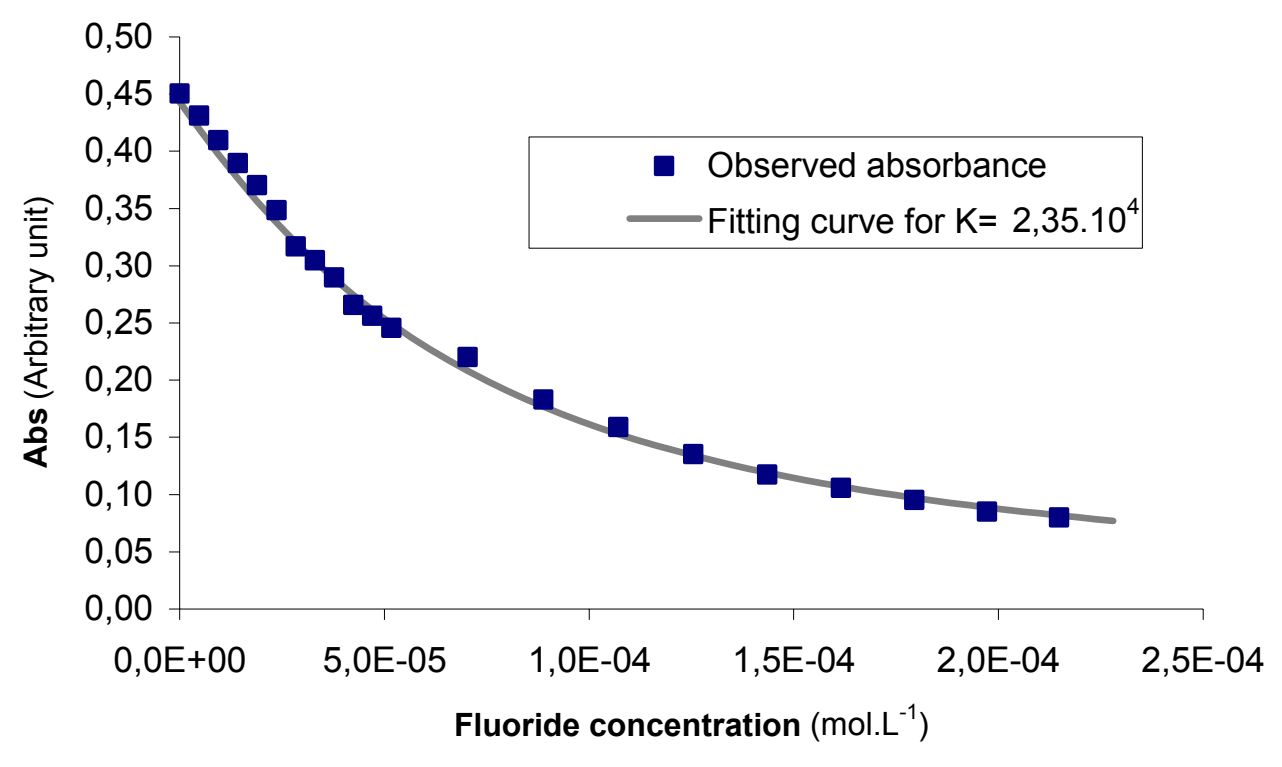

Figure SI- 4: Absorbance of a solution of 2 (THF- $\mathrm{H}_{2} \mathrm{O}$ : 90/10) vs. fluoride concentration.

The experimental data was fitted by hand to the above equation $\left(\mathrm{C}_{p F B H g 0}=4.510^{-5} \mathrm{M}\right)$. This fit yielded $\mathrm{K}_{p F B H g}=2.35( \pm 0.2) 10^{4} \mathrm{M}^{-1}$.

\section{$\underline{\text { Binding selectivity studies }}$}

Fluoride binding by 2 also occurs in the presence of other anions. This has been confirmed by monitoring the absorption, ${ }^{1} \mathrm{H}$ NMR, or ${ }^{19} \mathrm{~F}$ NMR spectra of 2 upon successive additions of different anions. For example, the successive addition of chloride, bromide, and iodide to a solution of $\mathbf{2}$ in THF does not lead to any noticeable changes of the spectroscopic features of $\mathbf{2}$. Addition of fluoride to the same solution results in the formation of $[2-\mathrm{F}]^{-}$. Similar experiments have been carried out with the other anions. 
Solid state luminescence spectrum of 2 and $\left[2-\mu^{2}-\mathrm{F}\right]^{-} \cdot\left[\mathrm{S}\left(\mathrm{NMe}_{2}\right)_{3}\right]^{+}$

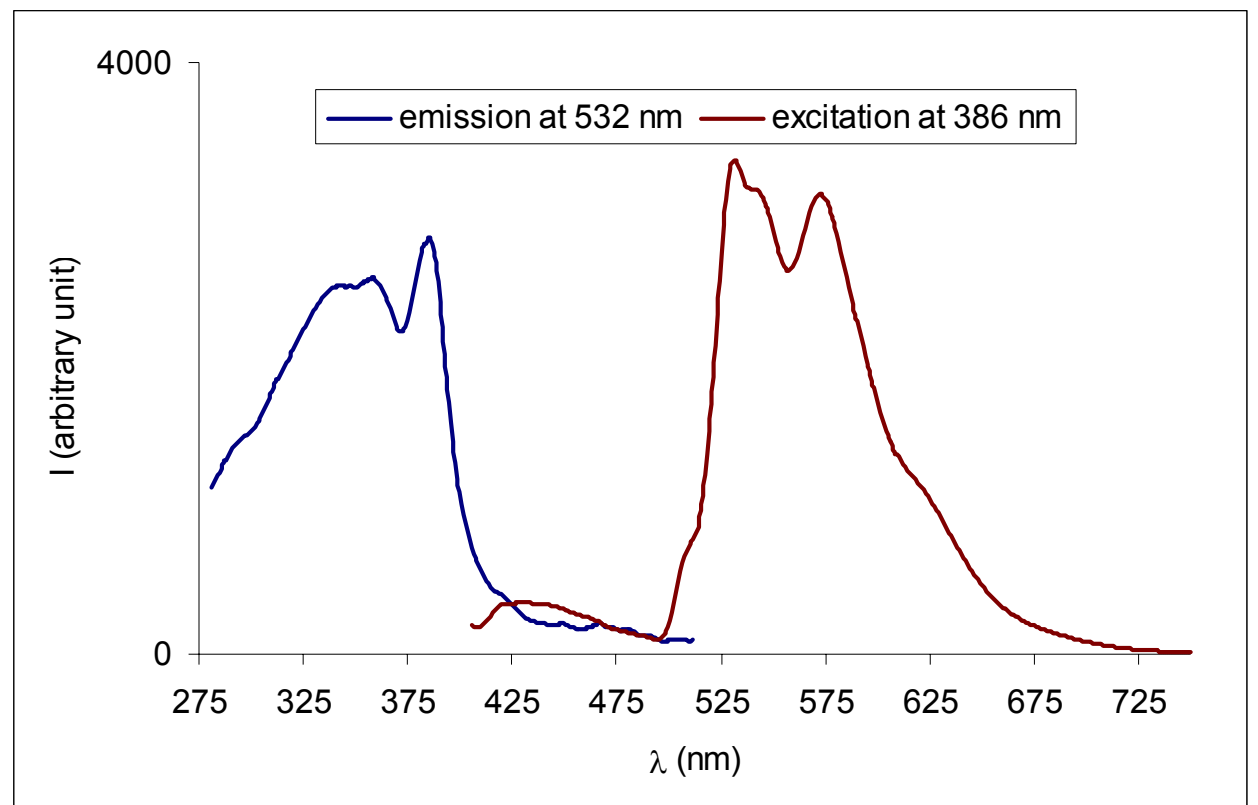

Figure SI- 5: Emission and excitation spectrum of 2 at $77 \mathrm{~K}$.

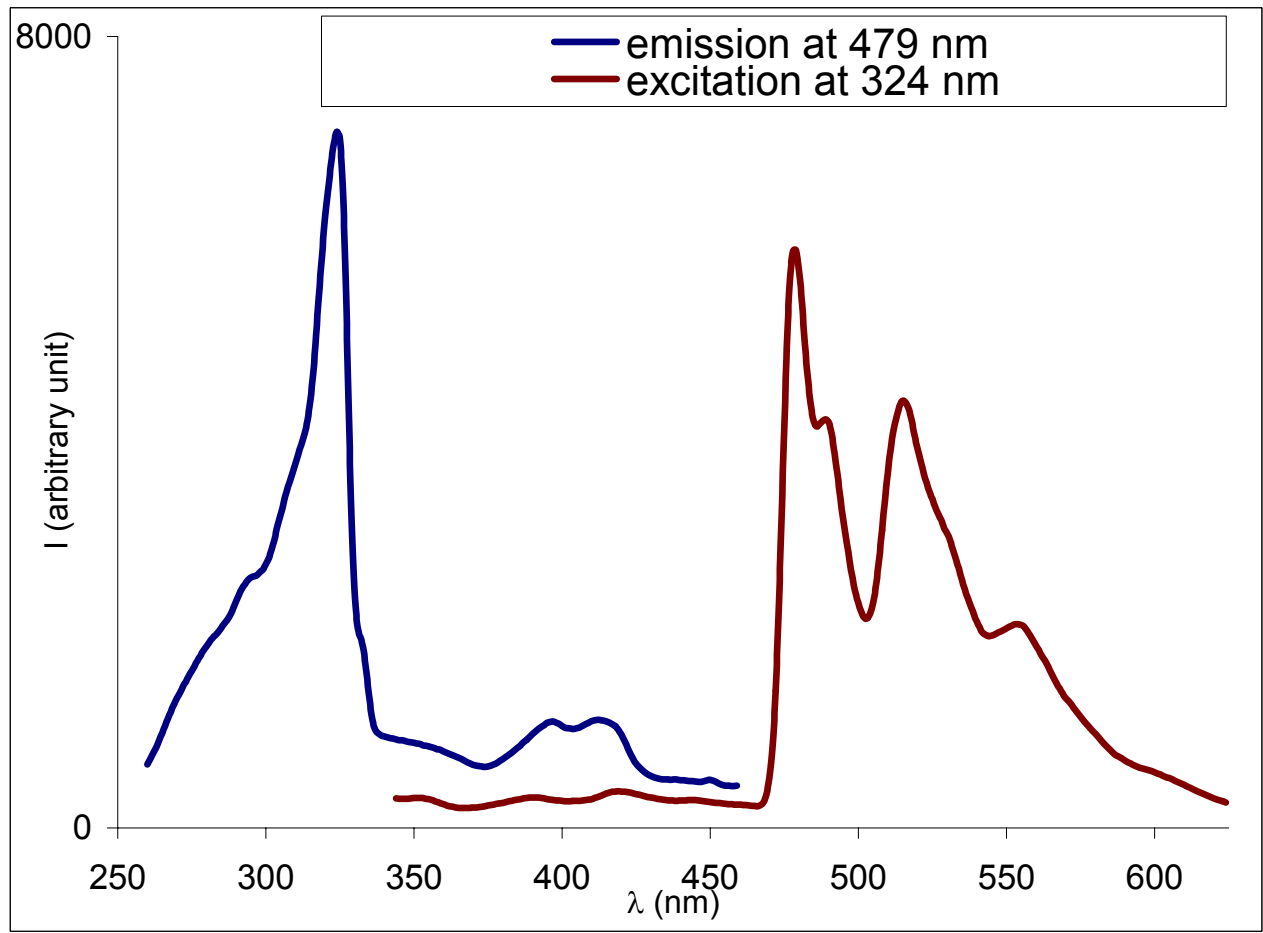

Figure SI- 6: Emission and excitation spectrum of $\left[2-\mu^{2}-\mathrm{F}\right]^{-} \cdot\left[\mathrm{S}\left(\mathrm{NMe}_{2}\right)_{3}\right]^{+}$at $77 \mathrm{~K}$. 